\title{
Julio Caro Baroja, antropólogo e historiador social
}

Si ya de por sí es complicado resumir en el poco espacio de unas páginas cualquier vida, en el caso de D. Julio Caro Baroja la tarea entraña una serie de importantes dificultades añadidas. Por empezar por los propios condicionantes personales, lo que seguramente no es en absoluto apropiado, las circunstancias en que se escribe esta semblanza para su homenaje, con la muerte de don Julio tan reciente y el sincero sentimiento por su pérdida todavía muy fresco, quizá no son las mejores para intentar objetivar una trayectoria intelectual e investigadora que aún nos resistimos a creer que haya tenido un punto final.

Entre estas circunstancias cuenta también la gran cantidad de escritos, homenajes, recuerdos que en muchos medios diferentes y de parte de personas igualmente diversas han aparecido y cuya lectura, hay que reconocer que con cierta dosis de subjetividad inevitable, a veces puede merecer un juicio negativo y otras veces dejar una sensación de insatisfacción. Porque ¿qué se puede decir de don Julio que no esté ya escrito por sus amigos más cercanos, y de mejor manera, por él en Los Baroja? ¿qué semblanza de sus inquietudes, sus modos, sus temas de trabajo, sus motivos puede ser más fiel que las Vidas Mágicas o sus cuadros? Someter así su vida a una forma resumida y casi seguro académica no puede crear más que intranquilidad.

Pero no es este el único, ni siquiera el más importante de los problemas. Lo fundamental es la riqueza extraordinaria y la enorme cobertura temática de una obra que por abarcar, supera los límites de varias disciplinas y afecta a numerosas especialidades dentro de ellas ¿Cómo resumir, sabiendo de qué se trata, trabajos de tecnología, lingüística, magia; sobre vascos, nómadas o judíos; de literatura, arquitectura, biografías, historia contemporánea, moderna, de las mentalidades, sobre mitología; acerca de Menandro o el doctor Torralba?

Junto a la variedad, y ligada a ella la cantidad, de unos escritos que forman una lista bibliográfica con casi setecientos títulos ${ }^{1}$, hay que apreciar

1 La bibliografía de Julio Caro Baroja es ciertamente compleja, no solo por su tamaño sino también por las modificaciones y reediciones que a lo largo del tiempo 
también la complejidad de la factura, y la lectura, de muchos de ellos. Si no es posible encasillar al autor en los estrechos límites de disciplinas, especialidades o asignaturas que se usan en los medios universitarios -algo sobre lo que además don Julio tenía un convencimiento y una voluntad radicalmente manifiestos- la elección de los temas de trabajo y la forma de desarrollarlos tampoco obedeció casi nunca a los cánones de la "academian.

En muchos casos Caro Baroja fue precursor, verdaderamente original, y no mero introductor de las corrientes impuestas en otros países con comunidades científicas más avanzadas. Repasando en su bibliografía los significativos años que corren entre 1957 y 1968 encontramos libros como Los moriscos del reino de Granada (1957), Las brujas y su mundo (1961), Los judios en la España moderna y contemporánea (1961-62), Vidas mágicas e Inquisición (1967), El señor inquisidor y otras vidas por oficio (1968), que nos sitúan en un momento de renovación del conocimiento histórico y social, pero del que a España no llegaban sino ecos lejanos y desvaídos. Éstas y otras obras constituyen no solo una excepción en la historiografía que se hacía por aquellos tiempos en España, sino también una real innovación dentro de las modernas ideas que se comenzaban a poner en práctica en la historia cultural y social (o historia antropológica después) fuera de nuestro país. La importancia dada a los individuos, a los casos particulares, a las biografías, a la percepción de las creencias, a las personalidades y los grupos marginales son ejemplo de innovación metodológica y muestran cómo la visión de la realidad histórica de Caro superaba los esquemas de la historiografía tradicional y se enlazaba en cambio con algunas de las más exitosas corrientes posteriores.

se han ido produciendo. Creo que todos tenemos con Antonio Carreira una deuda por haber asumido, además con tal rigor y minuciosidad, la tarea de ordenar y cuidar la obra de don Julio. Aparte de algunas recopilaciones restringidas anteriores (ver Carreira, 1994: 9), las bibliografías más completas de Caro se deben siempre a CARREIRA que publicó la primera de ellas (con 354 entradas) en 1978: CARREIRA, CID, GuTiÉrReZ y RUBIO (eds.), Homenaje a JCB (Madrid: Centro de Investigaciones Sociológicas), pp. 15-41. Posteriormente en 1982 (433 entradas): Homenaje a JCB del Club Cultura y Sociedad (Madrid: M. de Cultura), pp. 24-51; 1985 (510 entradas): Julio CARO y Emilio Temprano, Disquisiciones antropológicas (Madrid: Istmo), secc. I, pp. 455-492; 1986 (546 entradas): Revista Internacional de los Estudios Vascos, XXXI, pp. 247-290; además de una bibliografía esencial, incluyendo 80 fichas (1989): JCB Premio Nacional de las Letras Españolas 1985 (Barcelona: Anthropos/M. de Cultura), pp. 134-140, y un ensayo de clasificación temática (1994): Cuadernos Hispanoamericanos, 533-34, pp. 9-31. Una de sus últimas aportaciones (1995) recoge las 82 entradas de los estudios de Caro Baroja dedicados a Navarra: Príncipe de Viana, LVI, 206, pp. 569-575. En la RIEV, XI, 2 (1995), pp. 471-484, ha aparecido la última entrega de la bibliografía de Caro Baroja, desde 1985 hasta la actualidad. 
En buena medida es el carácter antropológico de esta obra histórica lo que la distingue. Si renovador fue el trabajo de Caro Baroja en lo que respecta a la investigación histórica, en el campo de la etnología su contribución es fundacional. También en esta área se movió con absoluta libertad, pero, en contrapartida, acompañada de una gran soledad. Su primera vocación intelectual fue la antropología, nacida de la curiosidad que le provocaba, y el contraste que representaba con su forma de vida habitual, la inmersión en el mundo campesino, arcaico y aislado de Vera de Bidasoa, donde los Baroja echaron de nuevo sus raíces vascas cuando Pío, el escritor, compró la casa de Itzea en 1912. El "Otro", lo "Otro" es lo que le atraía; evidentemente una motivación antropológica. Como muy propias de los antropólogos son también la crítica y la incomodidad con respecto a la propia sociedad a la que pertenecen, actitud vital igualmente clara en Julio Caro Baroja. Uno de los rasgos que recorren con más generalidad su obra - no solo la antropológica, también la histórica- es la incomodidad con las imágenes monolíticas, las teorías globalizadoras, los paradigmas establecidos; la negación a ver la realidad de una manera lineal, tópica o simplificadora. Por eso su interés en tratar de lo marginal, de lo que no es fácil de someter a la norma, lo variable; lo mismo da si se encuentra en la España del siglo XvII o en el desierto del Sáhara en 1953.

Sus primeras publicaciones son etnográficas y tempranísimas, ya que en 1929, es decir, contando el autor quince años de edad, se fecha "Algunas notas sobre la casa en la villa de Lesaka. Tanto éste como otros dos artículos posteriores, publicados en 1932 y 1933 en el Anuario de Eusko-Fol$k$ lore $^{2}$, nos muestran a un más que notable etnógrafo que conoce perfectamente el terreno, que emplea documentación histórica concreta para apoyar la observación directa, con una gran erudición y un conocimiento bibliográfico exhaustivo de lo publicado sobre el País Vasco y que, incluso, nos aporta con sus dibujos tomados del natural una visión detallada de las arquitecturas de que se ocupan los dos primeros trabajos. Estas obras iniciales obedecen a un plan de investigación ambicioso diseñado por José Miguel de Barandiarán en el Laboratorio de Etnología y Eusko-Folklore de la Sociedad de Estudios Vascos, con cuyos cuestionarios y bajo cuyas directrices Caro Baroja se inició en el estudio de la etnografía de los vascos. En 1934, cuando tenía veinte años, publica su primer libro: Tres estudios

2 Además del artículo sobre la arquitectura de Lesaka, Anuario de Eusko-Folklore, IX (1929), pp. 69-91, en la misma revista, dirigida por José Miguel de Barandiarán, aparecen «Monumentos religiosos de Lesaka", XII (1932), pp. 9-58 y "Cuatro relaciones sobre hechicería vasca», XIII (1933), 87-145. 
etnográficos relativos al país vasco; una rareza de la que se tiraron solo unos veinte ejemplares en la imprenta de su padre ${ }^{3}$.

No consistían, por tanto, en ningún entretenimiento de verano las campañas de campo y la colaboración con Telesforo de Aranzadi y José M. de Barandiarán. Un problema distinto era adquirir una completa formación en antropología en la universidad española, donde tales estudios no existían como especialidad independiente. En la Facultad de Filosofía y Letras de la Central de Madrid encontró, no obstante, algunos buenos maestros, entre ellos dos alemanes; Hugo Obermaier, con el que estudió prehistoria, y Hermann Trimborn, encargado de impartir etnología. La guerra civil cortó a Julio Caro Baroja la carrera por el medio, así que su paso por la universidad tampoco pudo ser nunca un recuerdo grato. En 1940 se licencia en Historia Antigua, terminando en un curso todas las asignaturas que le faltaban, y en 1942 recibe el título de doctor en la misma especialidad pero con una tesis sobre las fiestas ${ }^{4}$.

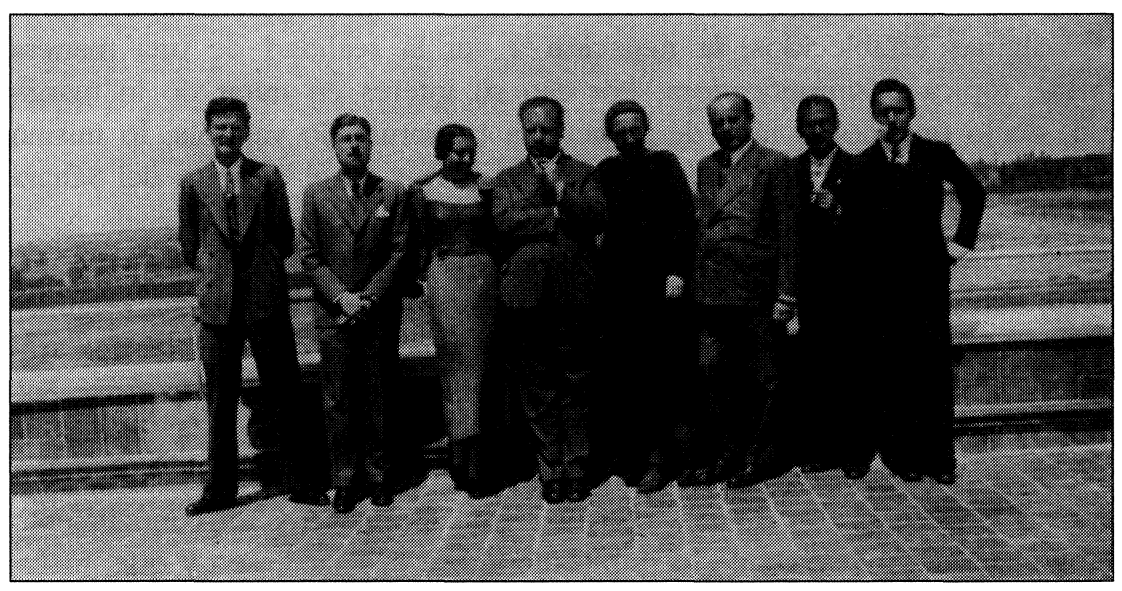

FIG. 1.-Caro Baroja (el primero de la izquierda) con sus compañeros de la Universidad Central de Madrid y su profesor de Etnología, Hermann Trimborn (en el centro).

3 Con prólogo de José Miguel de Barandiarán. Trabajos del Laboratorio de Etnología de la Sociedad de Estudios Vascos. Madrid, Imprenta de Caro Raggio, 1934, 125 pp. + figuras. Contiene tres trabajos: Sobre las lamias, Sobre arquitectura popular (el estudio sobre la arquitectura de Lesaka revisado) y Fragmentos de un estudio sobre particularidades etnográficas de la cuenca del Bidasoa (texto de una conferencia pronunciada en Vitoria en 1933). A. CARreira, "Bibliografía de D. Julio Caro Baroja", Homenaje a D. Julio Caro Baroja (Madrid: M. de Cultura, 1982), p. 25.

4 Viejos cultos y viejos ritos en el folklore de España. Tesis doctoral presentada el 7 de mayo de 1941 en la Facultad de Filosofía y Letras de la Universidad Central. CARREIRA, op. cit., 1982, p. 27. 
La influencia alemana de su etapa formativa es evidente, tanto del lado de sus maestros vascos, como de los profesores de Madrid, y se plasma muy claramente en el historicismo, la vinculación de la etnología y la prehistoria, y la adscripción a los postulados de la escuela difusionista austroalemana de sus primeros grandes libros: Los pueblos del Norte de la peninsula ibérica (Análisis bistórico cultural) (1943), Los pueblos de España. Ensayo de Etnología (1946), Análisis de la cultura (Etnologia-HistoriaFolklore) (1949), Los vascos. Etnología (1949) 5 .

A pesar de la conocida, y explícitamente manifiesta por él mismo muchas veces ${ }^{6}$, reticencia de Caro Baroja a incorporarse a las instituciones estatales de docencia e investigación de una forma "normal" $\multimap$ o viceversa, es decir, resistencia de las instituciones a incluirle a él- su primer cargo de responsabilidad administrativa es relativamente temprano. En 1944 es nombrado director del Museo del Pueblo Español, puesto donde permanece hasta 1955 en que, ante la imposibilidad de que el museo cumpliera sus mínimas funciones, presenta su dimisión. Esta década larga -que se superpone en parte a las fechas de los libros arriba citados- está muy caracterizada por su dedicación al museo; actividad que, ya que no pudo plasmarse en una exposición museográfica como hubiera sido deseable, se refleja con fuerza en su obra de investigación. Por un lado están los escritos dedicados a la catalogación de algunas colecciones pertenecientes a los fondos del Museo del Pueblo Español ${ }^{7}$ y a la exposición de sus teorías y proyectos museográficos para una gran colección etnográfica nacional ${ }^{8}$. Por otro, sus mejores artículos sobre tecnología tradicional datan de esta época 9 .

5 En el mismo sentido pueden citarse cinco recensiones de obras de autores alemanes, incluida una de Trimborn, debidas a Caro Baroja y que se publicaron en el órgano de difusión de la Sociedad Española de Antropología, Etnografía y Prehistoria, Atlantis, XVI (1941), pp. 478-485. Ver CARREIRA, op. cit., 1982, p. 26. Más adelante, entre los años 1949 y 1955, publicará otra serie de reseñas dedicadas esta vez a libros ingleses en el suplemento de la revista Insula. Ibid., pp. 29-31.

6 Ver por ejemplo Julio CARO BAROJA, "Una vida en tres actos", Homenaje a $D$. Julio Caro Baroja (Madrid: M. de Cultura, 1982), p. 17, donde se refiere a este tema de una forma muy concreta.

7 Aunque con un trabajo de mucha menor entidad, también colaboró en la catalogación de los fondos del Museo Etnológico, haciendo la descripción de los objetos procedentes de los grupos de moros de Joló y Mindanao para la Guía del Museo Etnológico (Madrid: CSIC, 1947), pp. 100-107.

8 Ver al respecto: Proyecto para una instalación al aire libre del Museo del Pueblo Español (Madrid: Trabajos y Materiales del Museo del Pueblo Español, s.a. [1948]) y aPosibilidades actuales y futuras del Museo del Pueblo Español, Homenaje a D. Mateo Silvela (Madrid, s.a. [1949]), 9-14. Estos y otros escritos sobre museografía de Caro Baroja se recopilaron en Museos imaginados (Gijón: Publicaciones del Museo Etnográfico de Grandas de Salime, 1986).

9 Una buena muestra se reunió en el libro Tecnología popular española (Madrid: Editora Nacional, 1983). Ver también los Cuadernos de campo (Madrid: Turner/M. de 
Pero además de lo que se refleja en la producción bibliográfica, hay en estos años otros aspectos muy importantes que seguramente fueron decisivos para la trayectoria intelectual posterior de Julio Caro Baroja y, sin duda, para su vida personal. En muchos aspectos, en la década de los cincuenta se definen una serie de cuestiones fundamentales. En lo personal, la muerte de su madre en 1950 y después de sus tíos Ricardo (1953) y Pío (1956) significaron para él una ruptura. En sus propias palabras

A los 42 años tuve la sensación de que otra gran etapa de la vida había terminado. Una etapa fuerte, intensa, con grandes dolores y grandes amistades, en que mi imagen del mundo se perfiló más.

¿Después? Después he pasado de la madurez a la senectud ${ }^{10}$.

A partir de los años sesenta parece haber cultivado esa imagen asumida de soltero, estudioso y solitario, y conformado su persona como el depositario de la herencia de un pasado, un entorno, una familia y un país desaparecidos, con la muerte de sus personajes, en sus mejores y más amables aspectos ${ }^{11}$.

Los cincuenta también suponen una ruptura en las líneas, los métodos y los temas de investigación. Si antes su círculo de observación y trabajo de campo preferente se situaba en el País Vasco, sus puestos administrativos en Madrid, en el Museo y en el Consejo Superior de Investigaciones Científicas (donde ya venía trabajando desde 1941, pero sobre todo desarrolla una gran actividad como secretario del Centro de Estudios de Etnología Peninsular a partir de 1947), le llevan a establecer contactos con otros colegas españoles y extranjeros, y le deparan oportunidades de estudio y de trabajo de campo en muy distintos terrenos.

Es así como, entre 1949 y 1950, realiza un largo viaje de reconocimiento cultural por toda España, pero sobre todo por el Sur, en compañía de George $M$. Foster, que recogía información para su proyecto de estudio comparado e influencias recíprocas entre España e Iberoamérica. Invitado por él, Caro Baroja viaja a Estados Unidos con una beca de la Wenner Gren Foundation para realizar estudios de antropología en la

Cultura, 1979). Sobre estos trabajos ver A. CARREIRA, «Los estudios tecnológicos de Caro Barojan, JCB Premio Nacional de las Letras Españolas 1985 (Barcelona: Anthropos/M. de Cultura, 1989), pp. 111-133.

10 Julio CARo BAROJA, «Una vida en tres actos", Homenaje a D. Julio Caro Baroja (Madrid: Ministerio de Cultura, 1982), p. 19. Este artículo fue antes publicado en la revista Triunfo (septiembre de 1981).

11 La imagen más precisa de la visión de su época y de sí mismo de Julio Caro Baroja se contiene en su libro de memorias, Los Baroja (Madrid: Taurus, 1972). 
Smithsonian Institution de Washington en 1951. Por medio de Foster había conocido en 1949 a Julian Pitt-Rivers, que se encontraba en Grazalema realizando su trabajo de campo y con el que don Julio estableció una sólida amistad. Pitt-Rivers fue el guía de otra estancia de estudios, está vez en Oxford, en el Institute of Social Anthropology, con una beca del British Council en 1952.

La frenética actividad desarrollada en estos años —en que, por otro lado, la muerte reciente de su madre, de su tío Ricardo y la postración en que se encontraba su tío Pío le sumían en la melancolía y la depresión-culmina con su trabajo etnográfico de campo más intenso y espectacular, el realizado entre los nómadas del Sahara Occidental en 1953. A instancias del director general de Marruecos y Colonias, José Díaz de Villegas, se plantea la realización de un trabajo etnográfico sobre los indígenas nómadas del entonces Sahara español, para el cual es recomendado Caro Baroja (seguramente por Tomás García Figueras), quien lleva a cabo la expedición en compañía de Miguel Molina Campuzano. Solamente tres meses permanecen en el territorio del Sahara Occidental y Río de Oro, pero el intensivo trabajo de campo, y el realizado antes y después del viaje sobre fuentes bibliográficas, producen un libro importantísimo dentro de la literatura antropológica nacional, los Estudios sabarianos (1955), y una serie de artículos que se recopilan en 1957 con el nombre de Estudios mogrebies ${ }^{12}$.

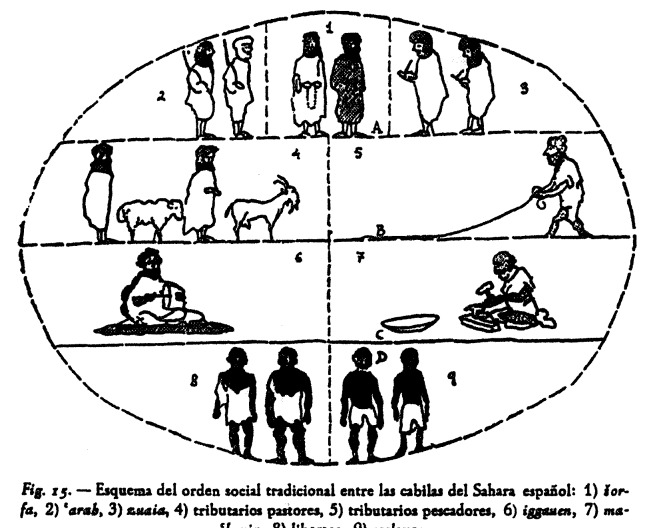

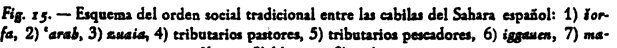
"lemin, 8) libertos, 9) exclavose

FIG. 2.-Esquema de distribución social. Dibujo de J. Caro Baroja. Estudios sabarianos, 1955 , p. 51.

12 Dentro del mismo grupo de publicaciones sobre el Norte de África debe contarse también un folleto titulado, Una visión de Marruecos a mediados del siglo XVI (la del primer historiador de los "xarifes", Diego de Torres) (Madrid: CSIC, 1956). Ver sobre estos trabajos, Mercedes GARCía ARENAL, "Estudios saharianos y mogrebíes", Cuadernos Hispanoamericanos, 533-34 (Homenaje a Julio Caro Baroja) (1994), pp. 209-216. 
El trabajo sobre los saharauis de Julio Caro Baroja resulta excepcional en varios sentidos. En primer lugar para su propio autor, que califica a los Estudios sabarianos "como el hijo intelectual más extraño de los muchos que he tenido" ${ }^{13}$, lo considera un enigma y siente por él un afecto especial. El enigma consiste en haber conseguido con tan poco tiempo una enorme cantidad de información especializada, en haber desarrollado una observación tan extensa y aguda sobre el terreno y, más importante aún, haberse sumergido tan profundamente y de una forma tan empática en una cultura alejada de la suya. El misterio, en fin, de los grandes etnógrafos de campo.

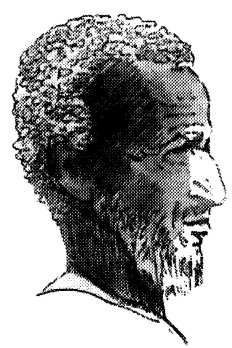

Fig. 78.-Otro tidrariní (tipo B)

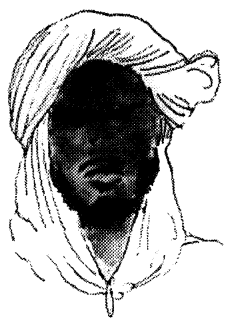

Fig. 80.-Otro tidrariní (tipo D) Fig. 81.-Un último tipo de tidarini (tipo E)

FIG. 3.-Tipos saharauis. Dibujos de Julio Caro Baroja. Estudios sabarianos, 1955.

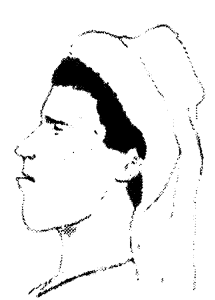

el tema de casi su último texto publicado sea el recuerdo de su viaje al Río de Oro ${ }^{15}$.

Por último, la excepcionalidad depende también del contexto en que debe situarse esta obra, dentro de

13 J. CARO BAROJA, «Prólogo», Estudios sabarianos (Gijón: Júcar, 1990, 2. a ed.).

14 Alfonso ARMADA, "Julio Caro Baroja salva el silencio de la otra orilla", El País, 12-XI-1995.

15 "Recuerdos de una estancia en el Sahara", Ciclo de conferencias de la Real Academia de la Historia (Madrid: Fundación Areces, 1993), pp. 13-26. Ver también "Los nómadas y su porvenir", Archivo de Estudios Africanos, XX, núm. 78 (1966), pp. 6171; "Un adiós a los nómadas saharianos", Blanco y Negro, núm. 3322 (3-I-1976), pp. 42-44 y aCaro Baroja y el pueblo Saharaui. Entrevista por Pedro Vaquero, Tiempo de Historia, núm. 65 (abril, 1980), pp. 108-113. 
una comunidad antropológica, la española, donde son contadas las ocasiones - y no consigue ninguna una categoría equiparable- en que el objeto de estudio lo constituye un pueblo distinto a los que forman nuestro propio país; es decir, donde la antropología se practica casi exclusivamente sobre los propios españoles. Leyendo los Estudios sabarianos se conoce no solo la vida, transcurrir histórico y carácter cultural de diversas cabilas de nómadas; se comprende también el modo particular como se desenvolvió el colonialismo africano en España y lo que, sin tópicos, éste dio de sí.

Volvamos ahora, tras este paréntesis del trabajo sahariano, al hilo cronológico que nos servía de conductor. Aunque para estas fechas sería absurdo hablar ya de formación, por muchas estancias de estudio en el extranjero que disfrutara, y a pesar de que él mismo ha expresado muchas veces que fue durante la guerra civil en que viví como el topo en su madriguera" ${ }^{16}$, cuando leyó, encerrado en la biblioteca de Vera, como

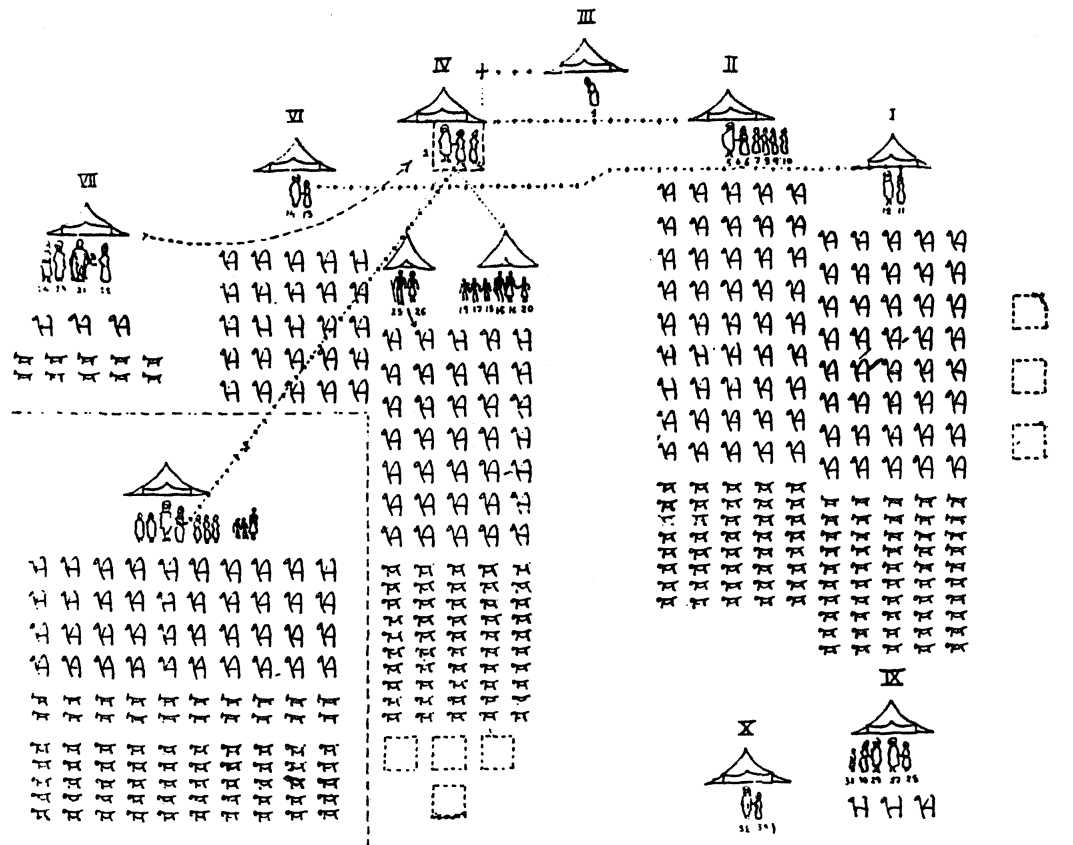

FIG. 4.-Distribución gráfica de las familias, y sus propiedades en ganado, de un campamento nómada. Dibujo del trabajo de campo en el Sahara. Estudios sabarianos, 1955, p. 205.

16 «Una vida en tres actos", op. cit., p. 15. 
luego ya nunca volvería a leer, es también cierto que sus estancias en Inglaterra y Estados Unidos determinaron no poco el enfoque y el método de sus trabajos posteriores.

Si en la década de los cuarenta la influencia alemana es patente en su trabajo antropológico, en los cincuenta y sesenta los intereses se relacionan más con el mundo anglosajón y con una idea funcionalista. La actitud manifiestamente historicista de Caro Baroja no podía ajustarse a la visión clásica del funcionalismo propuesta por Malinowski o RadcliffeBrown, hasta cierto punto estrecha, basada en la sincronía absoluta e inspirada en el modelo de sociedades relativamente primitivas y aisladas; con más propiedad lo que recogen sus trabajos es la crítica y el ajuste de la teoría funcional a sociedades complejas y con historia hechos por EvansPritchard ${ }^{17}$. Puede decirse sin duda que los Estudios sabarianos y mogrebies son las obras donde, de forma más patente, se hace una defensa explícita del método funcional. Pero hay que decir también que en ellas la historia, junto a la antropología social, aparece como un componente básico (incluso ocupando capítulos separados cada disciplina en Estudios sabarianos), y el historicismo (no del tipo morfológico, que interviniera en la escuela austroalemana, que es criticada) como un requisito perfectamente compatible con el funcionalismo antropológico.

A partir de esta época Julio Caro Baroja ha encontrado un método propio al que muchas veces, a partir de que emplee esta expresión por primera vez en su obra de mayor éxito, Las brujas y su mundo (1961), se referirá como "estructuralismo histórico" ${ }^{18}$. Pero, aún más importante que esto, ha encontrado la definición de la materia a la que se ajustan sus intereses como investigador: la historia social o, si queremos, la antropología histórica. La historia social es la disciplina a la que se dedican los antropólogos que trabajan sobre el desarrollo histórico de una sociedad. Es decir, como lo ha expuesto un especialista en la obra carobarojiana: "La historia social resulta ser, pues, una antropología de sociedades lejanas en el tiempo en lugar de una antropología de sociedades que son contemporáneas" ${ }^{19}$. Así pues, lo que la antropología social inglesa le aportó fue, paradójicamente, la clave para practicar sus estudios históricos.

Los años sesenta inauguran el periodo de madurez intelectual de Julio Caro Baroja. Como él mismo ha recordado más de una vez: "he escrito y

17 Francisco CASTILla URBANO, "El análisis histórico y antropológico de las minorías en la obra de Julio Caro Baroja", Cuadernos Hispanoamericanos, 533-34 (1994), pp. 83-98; p. 87.

18 F. Castilla Urbano, "Metodología en la obra de Julio Caro Baroja", Revista Internacional de los Estudios Vascos, XXXIV, 2 (1989), pp. 271-284; pp. 280-282.

19 F. CASTIlla URBano, "El análisis histórico y antropológico...", art. cit., p. 89. 
he publicado de 1960 a 1980 más que en el resto de mi vida" ${ }^{20}$. Pero además, la calidad de estos escritos es extraordinaria. La serie de los libros que consolidarán su prestigio internacional comienza con Los moriscos del reino de Granada (1957) ${ }^{21}$, sigue con Las brujas y su mundo (1961), sin duda su obra más conocida; Los judios en la España moderna y contemporánea (1961-62), El Carnaval (Análisis bistórico-cultural) (1965), Vidas mágicas e Inquisición (1967), Ensayo sobre la literatura de cordel (1969) y, ya unos años más tarde, Las formas complejas de la vida religiosa: Religión, sociedad y carácter en la España de los siglos XVI y XVII (1978); por citar solo algunos de los que obtuvieron mayor resonancia.

A pesar de la reiteración con que su autor, al menos en esta época, reclamaba para sí y para su trabajo - por muy diacrónicos o del pasado que fueran los temas tratados - la caraterización de "antropológico", estos libros, como otros muchos artículos y monografías, encontraron mayor eco entre los historiadores que en los medios antropológicos. Aparte de las reseñas y traducciones de algunas de sus obras, tal indicarían el nombramiento de Julio Caro Baroja, en 1960, como director de curso de la Sección de Historia Social y Económica de la École Pratique des Hautes Études de París y su entrada, en 1963, en la Real Academia de la Historia, respaldada su candidatura Por Ramón Menéndez Pidal, Manuel Gómez Moreno, Diego Angulo y Ramón Carande ${ }^{22}$.

Con todo, hay que recordar que el reconocimiento, aunque en estos años todavía más marginal, también se dio del lado de la comunidad antropológica internacional. A partir de la estancia en Oxford en 1952, la vinculación con el grupo de antropólogos formado en Inglaterra que, desde su primera dedicación africanista, comienza a proyectar su interés hacia Europa y concretamente hacia el Mediterráneo es algo más que coyuntural. Así, Caro Baroja participa en la conferencia que, patrocinada por la Wenner Gren, se celebra en 1959 en Burg Wartenstein (Austria), dirigida por Pitt-Rivers y con asistencia mayoritaria de especialistas británicos o que habían estudiado en Oxford, como Campbell, Peristiany, Abou-Zeid, Gellner, E. L. Peters y P. Stirling, pero a la que acuden también norteamericanos (E. Friedl o L. Wylie) y franceses (Bourdieu y Chiva) ${ }^{23}$. Prácticamente el

20 J. CARO BAROJA, "Una vida en tres actos", p. 20.

21 Ese mismo año publica Razas, pueblos y linajes (Madrid: Eds. de la Revista de Occidente), recopilación de artículos anteriores y alguno nuevo, que contiene uno de corte metodológico que expone los aspectos teóricos que se han tratado más arriba: «La investigación histórica y los métodos de la Etnología (Morfología y funcionalismo)", publicado antes en Revista de Estudios Políticos, 80 (1955), pp. 61-82.

22 J. CARO BAROJA, "Una vida en tres actos", p. 20.

23 Las aportaciones a este simposio fueron publicadas por PITT-RIVERs bajo el título de Mediterranean countrymen. Essays in the Social Antbropology of the Mediterra- 
mismo grupo, incluido el antropólogo español, bajo la dirección ahora de J. G. Peristiany, prosigue sus discusiones, centradas en los conceptos del honor y la vergüenza en las sociedades mediterráneas, en dos conferencias sucesivas, celebradas en 1961 y 1963 en Atenas. El resultado es de nuevo un libro de ensayos que puede considerarse fundacional en la que ha dado en llamarse Antropología del Mediterráneo: Honour and Shame. The Values of Mediterranean Society (1965) ${ }^{24}$. Por último, más de veinte años después, el reconocimiento de la comunidad antropológica se plasma en el nombramiento de Caro Baroja como miembro honorario del prestigioso Royal Anthropological Institute of Great Britain and Ireland.

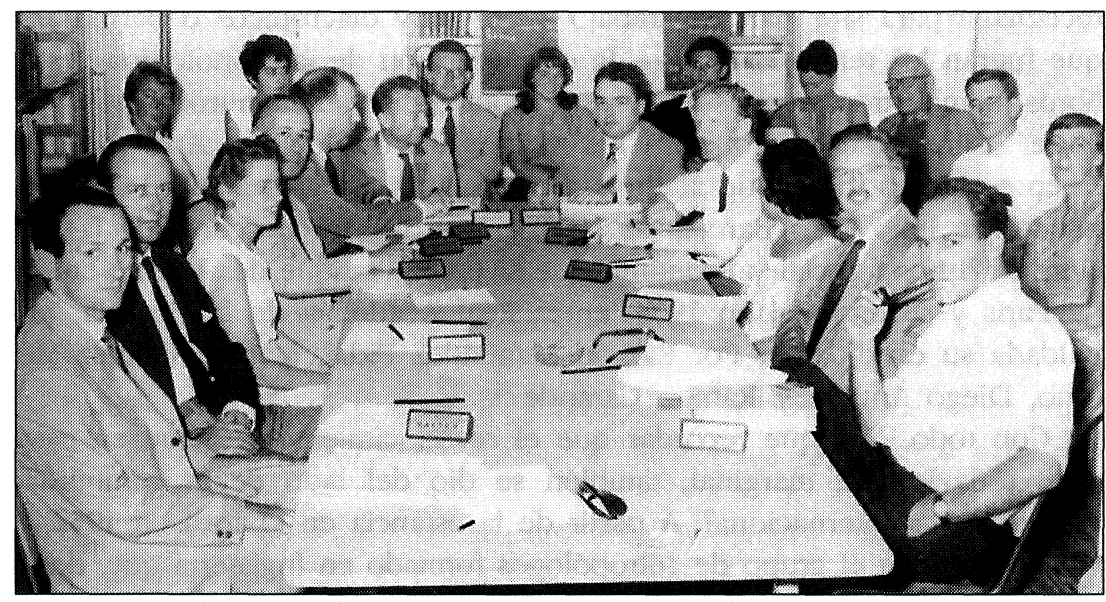

FIG. 5.-Conferencia antropológica celebrada en Atenas en 1963. Participan Kayser, Pitt-Rivers, Friedl, Peristiany, Wylie, Chiva, Bourdieu, Campbell, i ?, Caro Baroja y Gellner (en torno a la mesa, de izquierda a derecha).

Teniendo estos hechos a la vista resulta aun más sorprendente la frecuencia con que se le ha encasillado como un historiador atípico y aunque sea en menor medida como antropólogo, también atípico. En los años sesenta y dentro de su propio país se puede explicar que el gremio más receptivo ante la novedad que representaban sus obras tuviera que ser el de los historiadores, dada la falta de una mínima estructura profesional

nean (Paris-The Hague: Mouton, 1963). El artículo de Caro Baroja se titula "The City and the Country: Reflections on Some Ancient Commonplaces", pp. 27-40; se había publicado en español: «La ciudad y el campo o una discusión sobre viejos lugares comunes", en RDTP, XV (1959), pp. 381-400.

24 La contribución de J. CARO BAROJA es: "Honour and Shame: A Historical Account of Several Conflicts". La edición española del libro, El concepto del bonor en la sociedad mediterránea (Barcelona: Labor, 1968) lleva también un prefacio suyo. 
para la antropología. Sin embargo, cuando esta última circunstancia se produce de hecho, es decir, a partir de los años setenta, desde las nuevas cátedras y departamentos universitarios se insiste en la antropología del Mediterráneo, de los campesinos, de las sociedades complejas, pero se olvida el nombre y la aportación de Caro Baroja a esas mismas cuestiones para preferir como referencia otras figuras de su mismo grupo (los ingleses que primero acuden al Mediterráneo) o posteriores (algunos también de formación oxoniana como Carmelo Lisón) ${ }^{25}$. Es así significativo que, en estos años setenta, los dos textos que pueden citarse como mejor ejemplo de valoración de la contribución de Julio Caro Baroja a la antropología se deban ambos a reconocidos antropólogos anglosajones, PittRivers y Davydd Greenwood ${ }^{26}$.

No obstante, los años ochenta significan la llegada de un reconocimiento masivo por parte de la intelectualidad y de las instituciones culturales y científicas para la ya por entonces larga y fecundísima labor de don Julio. Suponen también su presencia pública en infinidad de foros, congresos, seminarios y eventos de todo tipo, así como una participación constante en los distintos medios periodísticos de comunicación. Sin embargo, su trabajo investigador obedece a unas líneas de continuidad y a unos objetivos concretos que se insertan en la concepción general que guía todos sus proyectos de estudio. De alguna manera, las publicaciones de Caro Baroja a partir de los setenta comienzan a tener un cierto carácter de recapitulación, en el sentido de que vuelve sobre viejos temas, recopila artículos dispersos para agruparlos en volúmenes de ensayos homogéneos o bien completa con obras nuevas aquéllas parcelas de su proyecto que aún quedan pendientes, como queriendo empezar a cerrar un círculo de intereses.

Acerca del mundo religioso y los conflictos que se producen en el ámbito de las creencias aparecen en estos años Inquisición, brujería y

${ }^{25}$ En este sentido uno de los juicios más improcedentes y desafortunados fue el de un recién doctorado en antropología que, en un artículo supuestamente historiográfico, mencionaba que la obra de Caro Baroja eno entra dentro de la esfera de las ciencias sociales». I. MORENO NAVARRO, "La antropología en Andalucía. Desarrollo histórico y estado actual de las investigaciones”, Ethnica, 1 (1971), 108-144; p. 130. Precisamente, un reconocido sociólogo, en un trabajo un poco posterior, hacía una valoración totalmente contraria, y muy elogiosa, de la labor sociológica de Caro: S. GINER, "Virtudes e indigencias de la sociología española", VV. AA., La cultura bajo el franquismo (Barcelona: Ediciones de Bolsillo, 1977), 133-155, p. 142.

26 Davydd J. GreENwoOD, "Julio Caro Baroja: sus obras e ideas", Ethnica, 2 (1971), pp. 79-97. Julian PITT-RIVERS, "A Personal Memoir", Homenaje a Julio Caro Baroja (Madrid: Centro de Investigaciones Sociológicas, 1978), pp. 887-893. 
criptojudaísmo (1970), De la superstición al ateísmo (Meditaciones antropológicas) (1974) e Introducción a una bistoria contemporánea del anticlericalismo español (1980). La trilogía sobre el ciclo anual festivo en la cultura popular, iniciada con El Carnaval, se completa con La estación de amor (Fiestas populares de mayo a San Juan) (1979) y El estio festivo (fiestas populares de verano) (1984). El campo y la ciudad, el paisaje y su interpretación cultural es otro motivo recurrente en estos años. Aunque ya había aparecido mucho antes, por ejemplo en La ciudad y el campo (1966), en los años ochenta se culmina una obra tan ambiciosa como La casa en Navarra (1982), aparecen varios artículos y libros de ensayos (Paisajes $y$ ciudades, 1984; Arte visoria, 1990), y hasta una muy personal guía cultural de Toledo (1988).

La biografía, no solo como género literario sino fundamentalmente como método válido de conocimiento antropológico e histórico, es otro de los motivos constantes que atraviesan el proyecto carobarojiano. En 1972 coincide la publicación de los dos libros fundamentales en este terreno, las Semblanzas ideales, una recopilación de retratos de personajes con los que el autor tuvo un contacto estrecho, y sobre todo sus impresionantes memorias familiares, Los Baroja. Pero en realidad todos sus libros están poblados de individuos, de personajes llamativos, marginales, falsarios, mesiánicos, además, por supuesto, de la gente corriente.

Si su obra autobiográfica y las semblanzas de sus maestros y amigos participan de un tono literario y de recreación personal de los recuerdos, sus biografías históricas, la mayoría ocupadas en personajes de los siglos XVI y XVII, transmiten una perspectiva diferente. La reconstrucción se basa en documentos escritos perfectamente contrastables y, a pesar del indudable atractivo de las acusadas personalidades de marginados o dominadores, en sí mismas, lo que se produce es una mirada voluntariamente distanciada que busca la explicación de ciertas formas arquetípicas de conducta, a partir de su práctica en casos concretos -es decir, las historias de vida de alguno de sus representantes-, y la relación de estas trayectorias individuales con la estructura social en la que se desenvuelven ${ }^{27}$.

La atención a la historia personal y la importancia otorgada a la escala de análisis proporcionada por el ciclo vital de los individuos es significativa de la renovación de las tendencias historiográficas a partir de los años setenta (historia de las mentalidades, de la vida cotidiana, etc.). También se ha indicado que este terreno de las historias de vida es uno de los mejores para que se produzca un acercamiento metodológico entre disciplinas cercanas pero separadas, como la historia, la antropología o la

27 F. CASTILla URBANO, "Sobre la 'Nueva Historia': autobiografía, biografía e historias de vida en la obra de Julio Caro Baroja», Antropología, 4-5 (1993), pp. 163-192. 
sociología, ya que en él la frecuente separación entre ciencias sociales y humanidades resulta en gran medida desdibujada por la comunidad del método y los objetivos ${ }^{28}$.

Pero además, en Caro Baroja, la práctica antropológica de trabajo de campo anterior, el proyecto de desarrollar un estructuralismo histórico y el interés teórico por los arquetipos y por descubrir los mecanismos internos en que se basa la producción de la propia documentación sobre la que luego se apoya la reconstrucción histórica, son las motivaciones que le llevan a elaborar sus estudios fundamentales en este género, recopilados ya en los años sesenta en libros como Vidas mágicas $e$ inquisición (1967) y El señor inquisidor y otras vidas por oficio (1968), a los que se suma después Vidas poco paralelas (con perdón de Plutarco) (1981).

Si todas sus biografías históricas son antropológicas, con independencia de la cronología concreta en que se desenvuelvan, una de ellas lo es más que el resto en un aspecto metodológico que la hace muy particular. Se trata de un trabajo publicado en los cincuenta, dedicado al seij $\mathrm{Ma}$ el Ainin $(1830-31 ; 1910)$, un santón y líder político sahariano ${ }^{29}$, que resulta esclarecedor de los objetivos científicos con que se emprende el relato de una vida. La unión de datos procedentes de fuentes documentales y bibliográficas (en las que contrasta el diferente trato dado al personaje según sea el autor francés, español o del Norte de África), con los relatos mantenidos por tradición oral entre sus descendientes y partidarios -algunos de ellos hijos del propio jeque-, de los que Caro Baroja recogió directamente la historia de vida, así como la visita a la ciudad de Smara fundada por el santón, podrían haber dado lugar a un relato novelesco, para el que se dan todos los ingredientes, incluida la aparición, en territorio tan exótico, de un submarino. Al final, la narración resulta tremendamente atractiva, pese a la reconocida resistencia del autor a introducir elementos literarios para atenerse al objetivo trazado: "Hacer ver el nexo de una personalidad vigorosa con un tipo de sociedad y de cultura y con un devenir histórico en momento crítico de conflictos y luchas" ${ }^{30}$.

De la importancia otorgada por Caro Baroja a la historia individual es buena muestra, finalmente, el tema escogido para su discurso de ingreso en la Real Academia Española: Género biográfico y conocimiento antropológico (1985), donde, con su personal forma, sintetiza sus intereses en este género.

28 Jorge BALÁN, "Introducción", J. Balán (ed.), Las historias de vida en ciencias sociales. Teoría y técnica (Buenos Aires: Nueva Visión, 1974), pp. 8-11.

29 "Un santón sahariano y su familia", Estudios sabarianos (Madrid: CSIC, 1955), pp. 285-335.

30 Op. cit., p. 334 . 
Los vascos son otro de los sujetos fundamentales de su obra. Sus trabajos monumentales sobre el País Vasco-Navarro incluyen desde los problemas del origen y evolución de la lengua (Materiales para una bistoria de la lengua vasca en su relación con la latina, 1946; Sobre la lengua vasca y el vascoiberismo, 1979), a monografías etnológicas tan completas como Los Vascos, o proyectos de abordaje total de la historia, la etnohistoria y la etnología como el que culmina en los tres volúmenes de Etnografía bistórica de Navarra (1971-72). No parece, pues, que pueda discutirse objetivamente que Caro Baroja sea el mayor y mejor estudioso de la cultura de los vascos; otra cosa es que haya resultado ser una personalidad difícil de encasillar con comodidad, y siguiendo un criterio localista, como el antropólogo vasco de los vascos. De hecho, en esta etapa que arranca a fines de los setenta, su propia comunidad de origen es motivo de reflexión para nuestro autor. En la recopilación de sus escritos en una serie de 19 volúmenes que, bajo el título general de Estudios vascos, comienza a editarse en 1973, no se evita el análisis de fenómenos políticos actuales, ni la crítica de la violencia que rodea la ideología nacionalista vasca, conflictos a los que se dedican el tomo XII de la serie, titulado El laberinto vasco (1984) y el XVI, Problemas vascos de ayer y boy (1986) ${ }^{31}$.

La falta de un soporte teórico y metodológico suficientemente explícito en sus trabajos es uno de los tópicos que más frecuentemente se repiten respecto a la investigación de Caro Baroja. Sin embargo, de la lectura (aunque no sea exhaustiva) de su obra no puede deducirse tal afirmación, a pesar de que el autor se manifieste muchas veces contrario al exceso de esquematismo y hasta el dogmatismo que conlleva encorsetarse bajo los abundantes "ismos" que se suceden con rapidez y que a menudo más que sustentarse en teorías potentes, suponen simplemente acogerse a una escuela. Por el contrario, en la enorme y variadísima obra que ha producido se observan sutiles hilos que conducen de unos problemas a otros y que dibujan un mundo de creación científica original.

Por otra parte, su preocupación por la teoría es constante, aunque a veces la resolución de los problemas de esta índole le plantee ciertas dudas y dificultades. En este sentido, algunos de sus libros se encaminan, a través de un exposición histórica de los principios que, sucesivamente, han regido el pensamiento antropológico, a mostrar la variedad de soluciones que han podido darse para las mismas cuestiones básicas y cómo, depen-

31 Ver Davydd GREENwOOD, "Etnicidad, identidad cultural y conflicto social: una visión general del pensamiento de Julio Caro Barojan, Julio Caro Baroja. Premio Nacional de las Letras Españolas 1985 (Barcelona: Anthropos/M. de Cultura, 1989), pp. 12-33, pp. 28-31. Al terrorismo dedica J. Caro Baroja otra monografía: Terror $y$ terrorismo (Barcelona: Plaza \& Janés / Cambio 16, 1989). 
diendo de muy diversos factores y condicionantes, unas han tenido mayor trascendencia que otras. En un libro pionero, Análisis de la cultura (Etnología-Historia-Folklore) (1949), se aborda por primera vez la exposición de los conceptos claves y la historia más reciente de la disciplina antropológica. Pero será en las últimas décadas de su vida cuando dedique de nuevo su esfuerzo a estos aspectos, sobre todo en dos libros: La aurora del pensamiento antropológico (La Antropología en los clásicos griegos y latinos) (1983) y Los fundamentos del pensamiento antropológico moderno (1985), a los que puede añadirse otro, más parcial, pero igualmente importante en el terreno al que nos referimos, La cara, espejo del alma. Historia de la fisiognómica (1987) ${ }^{32}$. Éste, junto a uno de los últimos libros de Caro Baroja, Las falsificaciones de la Historia (en relación con la de España) (1991), es indicativo, a la vez, de la curiosidad suscitada en el autor por los caminos, errados en el primer caso y falsos directamente en el segundo, por los cuales ha transitado muchas veces el conocimiento "científico" del hombre y de sus actos. Esta actitud está en

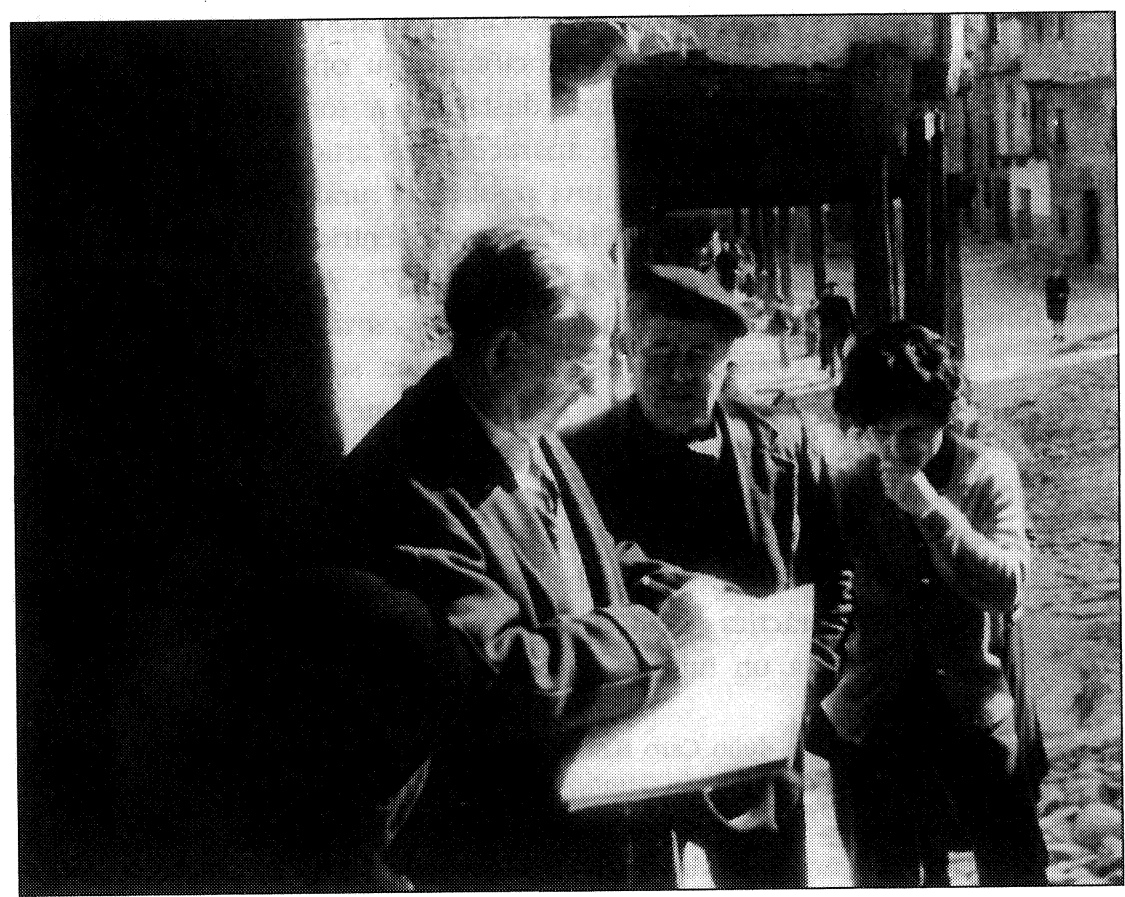

FIG. 6.-Julio Caro Baroja en un viaje de trabajo por Extremadura, 1973.

32 Ver sobre estos trabajos, Carmen ORTIZ GARCía, "Las historias de la antropología de Julio Caro Baroja", Cuadernos Hispanoamericanos, 533-34 (1994), pp. 55-67. 
relación con el respeto que cree debe guardarse a la concepción del conocimiento como un proceso acumulativo, al que han contribuido más fuerzas, y más diversas, de las que las modas teóricas y las novedades actuales parecen tener hoy exclusivamente en consideración.

No solo sus ensayos sobre historia del pensamiento científico inciden en este aspecto, sino que en general son las formas de construcción racional o pseudorracional de la realidad las que han sido objeto central de análisis, prácticamente en toda la obra de Caro Baroja. De este carácter participarían desde las cosmovisiones o visiones mágicas del mundo de muchos de los personajes o grupos marginados de los que se ocupa, a las ideas "racionales" o avanzadas, que se imponían para justificar la diferencia y las actuaciones sobre los diferentes; la utilización de la falsedad en la reconstrucción del pasado y de la identidad grupal o nacional, y sus motivos; o los mecanismos definitorios y de funcionamiento de arquetipos, tópicos y lugares comunes ${ }^{33}$.

Dentro de este interés general por los arquetipos y las formas de funcionamiento de las ideas colectivas, las caracterizaciones tópicas sobre las formas de ser de las personas, en función de su oficio, su lugar de origen, étnia o "raza", ocupan un lugar destacado y temprano en la bibliografía de Caro Baroja. Ya en 1951 aparecen dos artículos, "Sobre psicología étnica" e "Ideas y personas en una población rural", que son recogidos, con otros ensayos sobre ideas raciales y sociocéntricas, al final de la década, en el libro Razas, pueblos y linajes (1957). Además de esta obra, será fundamental otra recopilación, El mito del carácter nacional. Meditaciones a contrapelo (1970). En ella, junto a una serie de artículos sobre lugares comunes, tópicos repetidos o directamente mentiras que circulan en la investigación histórica ("Del cuaderno de notas de un historiador perplejo", se titula esta primera parte), se incluye un amplio ensayo que trata de una falsa verdad -el carácter nacional-, que ha tenido trascendencia científica y que la tiene considerable en la mentalidad colectiva, con consecuencias prácticas de todo tipo, incluidas las políticas; a la cual, Caro Baroja considera un mito y analiza como tal ${ }^{34}$.

33 F. CASTHLla Urbano, sJulio Caro Baroja: una aproximación a su obra antropológica e histórican, Revista d'Etnologia de Catalunya, e.p.

34 Ver de la misma época, "Superstición y ciencia" (1972) y «De nuevo sobre "caracteres étnicos': lo vasco y lo vizcaíno y ciertos tópicos literarios", publicados en $D e$ la superstición al ateismo (Meditaciones antropológicas) (Madrid: Taurus, 1974). Más recientes: De los arquetipos y leyendas. Dos tratados introductorios (Barcelona: Círculo de Lectores, 1989); «Tópico literario y caracterizaciones antropológicas: caracteres nacionales", Revista de Occidente, 56 (1986), pp. 91-103 y "Sobre los caracteres nacionales", Fragmentos italianos (Madrid: Istmo, 1992). 
En éstos, como en otros muchos de sus escritos (y en muy diversos aspectos que no podemos aquí abarcar), lo que sorprende es la modernidad de las cuestiones que plantean, incluso, si se permite la expresión, su "postmodernidad". La obra de Julio Caro Baroja, leída y releída hoy, sorprende, además de por la originalidad y potencia científica que revela, por su novedad constante. Fijándonos, ya casi con una perspectiva histórica que dan los años transcurridos, no hay prácticamente ningún tema, ningún interés puntual de la antropología social que se hace actualmente en nuestro país - desde los problemas de la etnicidad y la identidad, al análisis cognitivo de los discursos- que no encuentre en esa obra un antecedente, un punto de vista particular, una gran cantidad de datos y, en definitiva, una referencia inexcusable de autoridad.

Es una pérdida enorme que el filón de la creatividad de don Julio se haya al fin e irremediablemente acabado. Sería lamentable, y desde luego evitable, que nosotros no aprendiéramos algo, bastante, de todo eso que nos ha dejado.

Carmen OrTiz García

Departamento de Antropología Instituto de Filología. CSIC

Partiendo de la concepción de la obra de Julio Caro Baroja como un proyecto dotado de unidad interna, que tanto afecta a la historia como a la antropología, se analizan sus sucesivas influencias teóricas, se exponen sus principios teóricos y metodológicos y se reseñan algunas de sus aportaciones científicas en las temáticas concretas a las que dedicó su obra de investigación.

An analysis is made of the published works of Julio Caro Baroja, assuming that his whole project had an internal coherence and was related both to History and Anthropology. Other writers' influences on his work are scrutinized, as well as his theoretical and methodological background. Finally, some of his most important research contributions are summarized. 